\title{
Genetic identification of astroviruses in wild boars
}

\author{
Stefan Vilcek*, Slavomira Salamunova, Anna Jackova \\ Department of Epizootiology and Parasitology, University of Veterinary Medicine and Pharmacy, SK-041 81 Košice, Slovakia
}

\begin{abstract}
Astroviruses are widely detected in pigs but their detection in wild boars is rather sporadic. In this study, astroviruses were detected in organ homogenates of wild boars by applying nested reverse transcriptase polymerase chain reaction, and the typing was carried out by phylogenetic analysis. Overall, 30/200 (15.0\%) homogenates were positive for astroviruses. Genetic typing revealed that of 13 amplicons analyzed, 8 were typed as porcine astrovirus lineage 2 (PAstV-2), 2 as lineage 4 (PAstV-4), 2 identical sequences were grouped with chicken astrovirus, and 1 sequence belonged to a bat astrovirus lineage. This first identification of chicken and bat astroviruses in wild boars indicates interspecies transmission.
\end{abstract}

Keywords: Wild boar; Porcine astrovirus; Chicken astrovirus; Bat astrovirus; Phylogenetic analysis

Astroviruses as primary enteropathogenic agents belonging to the family Astroviridae, which is comprised of two genera, Mamastrovirus and Avastrovirus. The virions are small (28-30 $\mathrm{nm}$ ), non-enveloped, and contain a single-stranded, positivesense RNA molecule of 6.4 to $7.7 \mathrm{~kb}$. The genome is arranged in three open reading frames (ORF) with ORF1a and ORF1b at the $5^{\prime}$ end that encodes non-structural proteins and ORF2 at the 3 ' end that encodes structural proteins [7]. Astroviruses infect a wide range of mammalian species including humans, farm animals (pigs, cattle, sheep), companion animals (cats, dogs), as well as many wild and aquatic mammalian species and birds [14]. Astrovirus infections in mammals are usually associated with gastroenteritis.

Porcine astroviruses (PAstVs) isolated from pigs worldwide, including several Central European countries, have been typed into five highly genetic variable lineages (PAstV-1 to PAstV-5) $[1,4,15]$. Viruses are mostly detected in enteric samples, but recently, PAstV was also found in the bloodstream of pigs [1], indicating a more complex pathogenesis of the virus. Information on astroviruses in wild boars is rather sporadic. Recently astroviruses have been confirmed present in fecal samples of wild boars (Sus scrofa) in Hungary [10] and South Korea [9].

In our laboratory, we have detected PAstV in over $90 \%$ of tested rectal swabs of diarrheic and healthy pigs in Slovakia. The detected astroviruses fall into all 5 genetic lineages identified for PAstVs so far [11]. The high frequency of detection and the genetic variability of astroviruses in domestic pigs inspired our investigation of these viruses in genetically related animals. The aim of this work was to investigate the genetic diversity of astroviruses infecting wild boars.

To detect astroviruses, tissue homogenates, prepared as a pool of spleen, kidney and lymph nodes, from 200 hunted wild boars were used in the study. The three organs had been collected from 100 juvenile boars ( $<1$ year old) and 100 mature wild boars ( $>1$ year old) from different regions of Slovakia in 2012. They were submitted by hunters to the Veterinary Institute in Zvolen, Slovakia as a part of a national classical swine fever virus surveillance program undertaken according to OIE rules. Prior to use, the homogenates had been stored at $-70^{\circ} \mathrm{C}$ and were kindly provided by Miroslav Mojzis, DVM, Zvolen, Slovakia. All pooled homogenates were negative for classical swine fever virus.

Total RNA was isolated by applying the Trizol method and synthesis of cDNA was carried out using random primers and RevertAid Premium reverse transcriptase (Thermo Scientific, USA). The polymerase chain reaction (PCR) primers panAV-F11 (forward), panAV-F12 (forward), and panAV-R1 (reverse) were selected from the RNA-dependent RNA polymerase (RdRp) gene in ORF1b. The primers panAV-F21 (forward), panAV-F22 (forward), and panAV-R1 (reverse) were used in semi-nested PCR which amplified a 407 to 413 bp DNA product of PAstV [2]. This type of PCR, along with above-mentioned primers, can detect a wide spectrum of astroviruses in many hosts,

Received 10 Oct. 2018, Revised 8 Nov. 2018, Accepted 18 Nov. 2018

*Corresponding author: Tel: +421-915-984654; E-mail: vilcek@uvm.sk 
including pigs, birds, and bats. Details of the procedure for the synthesis of cDNA and determining the composition of PCR mixture were similar to those for the detection of astroviruses in domestic pigs and described in our previous work [11].

To analyze the detected astroviruses at the genetic level, PCR amplicons $(\mathrm{n}=13)$ from PAstV-positive samples were used for sequencing and phylogenetic analysis. Selection of PCR amplicons was carried out on the basis of their quality and to cover the entire geographic area. Purification of PCR amplicons and sequencing in both directions using Sanger's method employing inner panAV-F21 (forward), panAV-F22 (forward), panAV-R1, and fluorescently labeled dideoxynucleotides was carried out by a commercial company Microsynth Austria (Austria). In our laboratory, the chromatograms were checked and edited by using the computer program SeqMan (Lasergene DNASTAR, USA). The nucleotide sequences were deposited in GenBank accession Nos. MH511524 to MH511538. The phylogenetic tree was constructed by the neighbor-joining method using the Kimura-2 parameter in the software program MEGA6 [13]

The PCR-positive samples for astrovirus sequences provided an electrophoretic band visible at position $410 \mathrm{bp}$. A total of 30 $(15.0 \%)$ positive samples were detected among the collection of 200 wild boar samples. Fifteen juvenile and 15 mature wild boars were identified as infected by astroviruses. In a previous study using metagenomic analysis, PAstV was detected in 5 of 10 fecal samples from wild boars in Hungary [10]. On the other hand, only 1 of 146 fecal samples of wild boar in South Korea was positive for PAstV [9]. However, it should be noted that the astroviruses mentioned in those two published papers were identified in fecal samples, whereas the samples in the present study originated from parenchymatic organs and/or lymph nodes.

Genetic analysis of selected PCR amplicons (Fig. 1) revealed that 8 boars were infected with PAstV-2 and 2 boars with PAstV-4. The PAstV found in wild boars in Hungary [10] and South Korea [9] were both typed as representative of the PAstV-4 lineage. For comparison, domestic pigs have yielded all five PAstV lineages [1,11]; it is likely that additional PAstV lineages will be detected in wild boars in the future.

Two identical astrovirus sequences $(2761,2774)$ detected in wild boars were identified as chicken astrovirus (CAstV-2; Fig. 1). Both virus-positive wild boars originated from distinct geographic regions of Slovakia. The origin of the chicken astrovirus in the wild boars is unclear. We speculate that this astrovirus may have been of dietary origin or the result of some direct/indirect contact between wild boars and birds. To test this hypothesis, we selected chicken and pheasants as possible sources of chicken astrovirus. We analyzed rectal swabs of 10 hens from Slovakia for astrovirus presence. All birds were positive for astrovirus and had identical nucleotide sequences (data not shown). Genetic analysis confirmed that the astrovirus

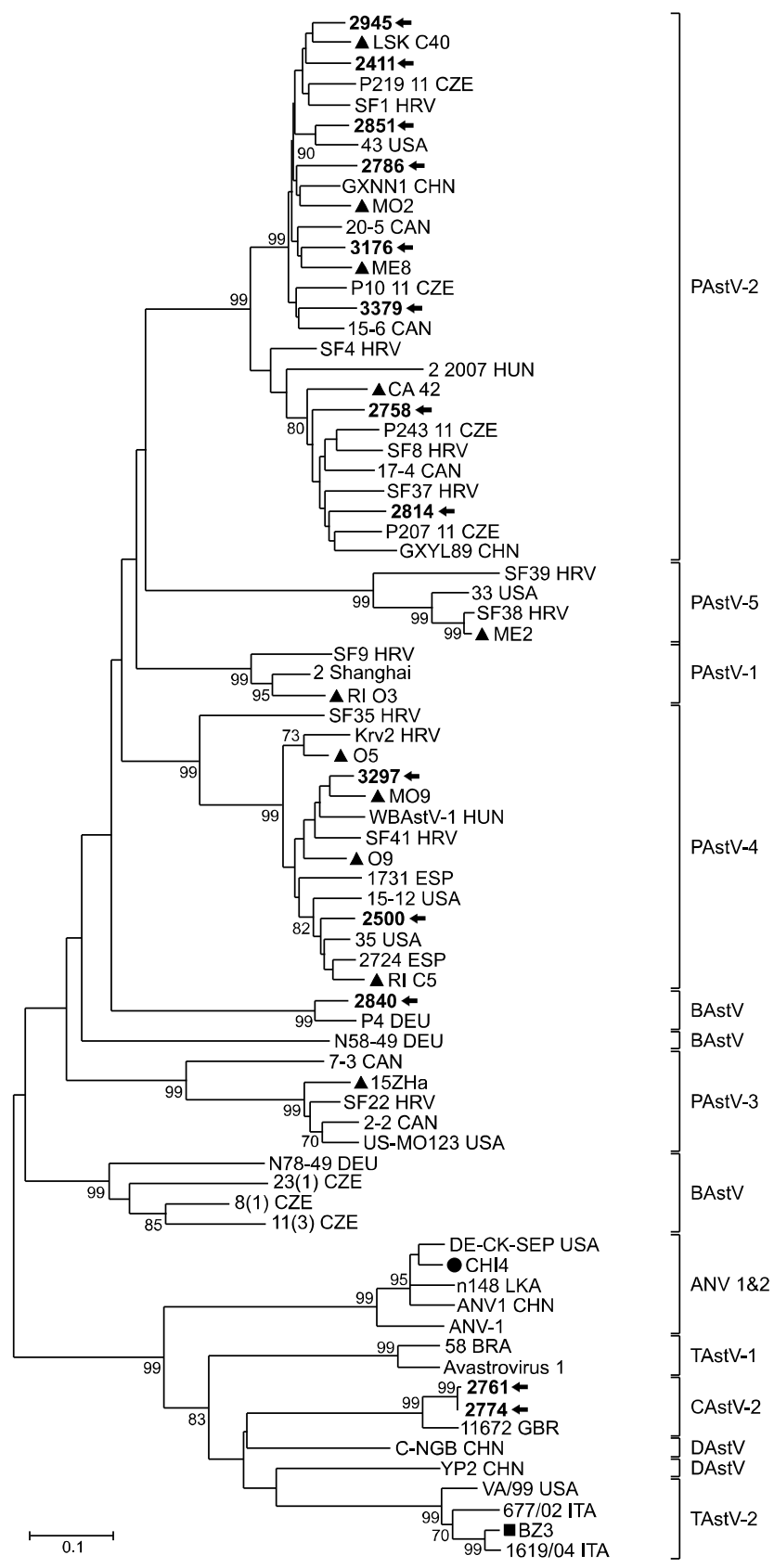

Fig. 1. Phylogenetic tree of astroviruses. The tree was constructed from a 317 to 347 bp DNA fragment (primers were omitted) depending on the astrovirus nucleotide sequences of the RNA-dependent RNA polymerase (RdRp) gene by a neighbor-joining method incorporated in MEGA6 [13]. Sequences from Slovakian wild boar samples are in bold with arrows, sequences from domestic pigs in Slovakia are marked with a triangle, while those from chickens are marked with a black circle and from pheasants with a black cube. Selected sequences from GenBank are labeled with their country of origin and accession numbers. The scale bar represents the number of nucleotide substitutions per site. PAstV, porcine astrovirus; CAstV, chicken astrovirus; TAstV, Turkey astrovirus; BAstV, bat astrovirus; DAstV, duck astrovirus; ANV, avian nephritis virus. 
sequence from our hens (CHI4) was grouped with avian nephritis virus type $1 \& 2$ (ANV 1\&2; Fig. 1). We also undertook detection of astrovirus in 21 rectal swabs from pheasants, and 5 of those were astrovirus-positive. Genetic analysis of the PCR product revealed that the pheasant astrovirus sequence (BZ3) was grouped with a turkey astrovirus (TAstV-2; Fig. 1), and the sequence was not genetically related to astroviruses found in wild boars or chickens. Nevertheless, to the best of our knowledge, we have identified for the first time that pheasants are also infected by astrovirus, thereby extending the spectrum of bird species to be infected by this virus.

Several authors have observed that avian astroviruses do not strictly infect avian species only. For example, Avastrovirus has been detected in diarrheic mink in China [12] and in feces from cats in Florida [8]. In addition, non-human primates can harbor a wide spectrum of mammalian and avian astroviruses [6]. Our study has confirmed that chicken astrovirus can be detected in wild boars.

Surprisingly, the nucleotide sequence of sample 2840 fell into the phylogenetic branch of the bat astrovirus (BAstV) cluster (Fig. 1). This sequence was grouped together with a nucleotide sequence that was detected in a saliva sample from Plecotus auritus (isolate P4; Fig. 1) in Germany in 2011. Thus, we have identified for the first time a bat astrovirus in a wild boar, but the origin of this astrovirus in the boar is unknown. All other bat astrovirus sequences previously identified in the Czech Republic [5] and Germany (Fig. 1), as well as in Hungary (data not shown due to short nucleotide sequences available in GenBank), were not related to the bat astrovirus identified in the wild boar in Slovakia. A comprehensive phylogenetic analysis has shown that some sequences found in bats do cluster with astrovirus strains from other species, including fox, cattle, and mice [3], an observation suggesting that bats could be involved in the interspecies transmission of astroviruses.

In summary, of the five known PAstV lineages, lineages PAstV-2 and PAstV-4 were identified in wild boar samples from Slovakia, thereby elucidating the genetic diversity of detected PAstVs in wild boars. In addition to PAstV, two identical chicken astrovirus sequences and one bat astrovirus sequence were detected for the first time in wild boars. Our observations indicate that astroviruses are able to undergo interspecies viral transmission in wild boars and other animals.

\section{Acknowledgments}

We would like to thank Peter Nettleton, Edinburgh for critical reading of the manuscript and correction of English grammar. This work was supported by projects APVV-15-0415 from The Slovak Research and Development Agency and Medical University Park in Košice (MediPark, Košice) ITMS: 26220220185 supported by Operational Programme Research and Development (OP VaV-2012/2.2/08-RO) (contract No.
OPVaV/12/2013).

\section{Conflict of Interest}

The authors declare no conflicts of interest.

\section{References}

1. Bmić D, Jemeršić L, Keros T, Prpić J. High prevalence and genetic heterogeneity of porcine astroviruses in domestic pigs. Vet J 2014, 202, 390-392.

2. Chu DK, Poon LL, Guan Y, Peiris JS. Novel astroviruses in insectivorous bats. J Virol 2008, 82, 9107-9114.

3. Donato C, Vijaykrishna D. The broad host range and genetic diversity of mammalian and avian astroviruses. Viruses 2017, 9, 102.

4. Dufkova L, Scigalkova I, Moutelikova R, Malenovska H, Prodelalova J. Genetic diversity of porcine sapoviruses, kobuviruses, and astroviruses in asymptomatic pigs: an emerging new sapovirus GIII genotype. Arch Virol 2013, 158, 549-558.

5. Dufkova L, Straková P, Širmarová J, Salát J, Moutelíková R, Chrudimský T, Bartonička T, Nowotny N, Růžek D. Detection of diverse novel bat astrovirus sequences in the Czech Republic. Vector Borne Zoonotic Dis 2015, 15, 518-521.

6. Karlsson EA, Small CT, Freiden P, Feeroz MM, Matsen FA 4th, San S, Hasan MK, Wang D, Jones-Engel L, Schultz-Cherry S. Non-human primates harbor diverse mammalian and avian astroviruses including those associated with human infections. PLoS Pathog 2015, 11, e1005225.

7. King AMQ, Adams MJ, Carstens EB, Lefkowitz EJ. Virus Taxonomy: Classification and Nomenclature of Viruses: Ninth Report of the International Committee on Taxonomy of Viruses. Elsevier, Oxford, 2012.

8. Lawler PE, Cook KA, Williams HG, Archer LL, Schaedel KE, Isaza NM, Wellehan JFX Jr. Determination of the diversity of astroviruses in feces from cats in Florida. J Vet Diagn Invest 2018, 30, 275-279.

9. Lee MH, Jeoung HY, Park HR, Lim JA, Song JY, An DJ. Phylogenetic analysis of porcine astrovirus in domestic pigs and wild boars in South Korea. Virus Genes 2013, 46, 175-181.

10. Reuter G, Nemes C, Boros A, Kapusinszky B, Delwart E, Pankovics P. Astrovirus in wild boars (Sus scrofa) in Hungary. Arch Virol 2012, 157, 1143-1147.

11. Salamunova S, Jackova A, Mandelik R, Novotny J, Vlasakova M, Vilcek S. Molecular detection of enteric viruses and the genetic characterization of porcine astroviruses and sapoviruses in domestic pigs from Slovakian farms. BMC Vet Res 2018, 14, 313.

12. Sun N, Yang Y, Wang GS, Shao XQ, Zhang SQ, Wang FX, Tan B, Tian FL, Cheng SP, Wen YJ. Detection and characterization of avastrovirus associated with diarrhea isolated from minks in China. Food Environ Virol 2014, 6, 169-174.

13. Tamura K, Stecher G, Peterson D, Filipski A, Kumar S. MEGA6: Molecular Evolutionary Genetics Analysis 
version 6.0. Mol Biol Evol 2013, 30, 2725-2729.

14. Vu DL, Bosch A, Pintó RM, Guix S. Epidemiology of classic and novel human astrovirus: gastroenteritis and beyond. Viruses 2017, 9, 33.

15. Zhou W, Ullman K, Chowdry V, Reining M, Benyeda Z,
Baule C, Juremalm M, Wallgren P, Schwarz L, Zhou E, Pedrero SP, Hennig-Pauka I, Segales J, Liu L. Molecular investigations on the prevalence and viral load of enteric viruses in pigs from five European countries. Vet Microbiol 2016, 182, 75-81. 